\title{
Tissue Carnitine Homeostasis in Very-Long-Chain Acyl-CoA Dehydrogenase-Deficient Mice
}

\author{
UTE SPIEKERKOETTER, CHONAN TOKUNAGA, UDO WENDEL, ERTAN MAYATEPEK, \\ LODEWIJK IJLST, FREDERIC M. VAZ, NAOMI VAN VLIES, HENK OVERMARS, \\ MARINUS DURAN, FRITS A. WIJBURG, RONALD J. WANDERS, AND ARNOLD W. STRAUSS \\ Department of General Pediatrics [U.S., U.W., E.M.], University Children's Hospital, 40225 Düsseldorf, \\ Germany; Department of Pediatrics [U.S., C.T., A.W.S.], Vanderbilt Children's Hospital, Vanderbilt \\ University, Nashville, Tennessee 37232; and Departments of Pediatrics and Clinical Chemistry [U.S., L.I., \\ F.M.V., N.v.V., H.O., M.D., F.A.W., R.J.W.], University of Amsterdam, Academic Medical Center, 1105 \\ AZ Amsterdam, The Netherlands
}

\begin{tabular}{|c|c|}
\hline \multicolumn{2}{|c|}{ ABSTRACT } \\
\hline $\begin{array}{l}\text { Deficiency of very-long-chain acyl-CoA dehydrogenase } \\
\text { (VLCAD) is the most common long-chain fatty acid oxidation } \\
\text { defect and presents with heterogeneous clinical manifestations. Ac- } \\
\text { cumulation of long-chain acylcarnitines and deficiency of free car- } \\
\text { nitine have often been proposed to play an important role in disease } \\
\text { pathogenesis. The VLCAD-deficient mouse exhibits similar clinical } \\
\text { and biochemical phenotypes to those observed in humans and, } \\
\text { therefore, represents an excellent model to study VLCAD defi- } \\
\text { ciency. We measured carnitine and acylcarnitine profiles in liver, } \\
\text { skeletal muscle (SkM), bile, and blood from VLCAD knock-out } \\
\text { mice and controls under nonstressed and various stress conditions. } \\
\text { Carnitine and acylcarnitines were extracted from body fluids with } \\
\text { methanol and from tissues with acetonitrile, respectively, and were } \\
\text { analyzed as their butyl esters using electrospray ionization tandem } \\
\text { mass spectrometry. Fasting combined with a cold challenge for } 8 \mathrm{~h} \\
\text { significantly induced liver long-chain acylcarnitine and free carni- } \\
\text { tine production. Acylcarnitines in SkM predominantly accumulated } \\
\text { during exercise with a concomitant decrease of free carnitine. } \\
\text { Changes in blood free carnitine did not correlate with carnitine }\end{array}$ & $\begin{array}{l}\text { homeostasis in liver and SkM. Our results demonstrate different } \\
\text { tissue-specific long-chain acylcarnitine profiles in response to vari- } \\
\text { ous stressors, which may be of importance with respect to the } \\
\text { heterogeneous clinical manifestations of VLCAD deficiency in } \\
\text { humans. Furthermore, we conclude that carnitine biosynthesis in the } \\
\text { liver seems sufficiently active to maintain liver carnitine levels } \\
\text { during increased demand. Our data suggest that carnitine supple- } \\
\text { mentation in long-chain } \beta \text {-oxidation defects may not be required, } \\
\text { and blood carnitine concentrations do not reflect tissue carnitine } \\
\text { homeostasis. (Pediatr Res 57: 760-764, 2005) } \\
\text { HZ, heterozygous } \\
\text { KO, knock-out } \\
\text { SkM, skeletal muscle } \\
\text { VLCAD, very-long-chain acyl-CoA dehydrogenase } \\
\text { VLCADD, very-long-chain acyl-CoA dehydrogenase } \\
\text { deficiency } \\
\text { WT, wild-type }\end{array}$ \\
\hline
\end{tabular}

Deficiency of very-long-chain acyl-CoA dehydrogenase (VLCAD) is the most common mitochondrial $\beta$-oxidation defect of long-chain fatty acids, with an incidence of $\sim 1: 50,000$ to $1: 100,000$ births (1). In humans, three distinct clinical phenotypes of different severities occur: a life-threatening, early-onset presentation with cardiomyopathy and hepatopathy; a hepatic phenotype with recurrent hypoketotic hypoglycemia and onset in infancy; and a milder, myopathic form with episodic muscle weakness, myalgia, and rhabdomyolysis presenting in adolescence or adulthood (2). Treatment interven-

Received June 16, 2004; accepted September 27, 2004.

Correspondence: Ute Spiekerkoetter, M.D., Department of General Pediatrics, University Children's Hospital, Moorenstrasse 5, D-40225 Düsseldorf, Germany; e-mail: ute.spiekerkoetter@uni-duesseldorf.de.

DOI: 10.1203/01.PDR.0000157915.26049.47 tions such as avoidance of fasting, reduced long-chain fat intake, and supplementation with medium-chain triglycerides may avoid metabolic decompensation, and long-term rehabilitation can be achieved $(3,4)$.

Characteristically, long-chain acyl-CoAs accumulate in mitochondria. To leave the mitochondria, they are converted into acylcarnitine esters, which can be assayed in blood (5). As a result of an increased production of acylcarnitines, blood free carnitine concentrations may decrease (5), a condition termed secondary carnitine deficiency. Nevertheless, supplementation of exogenous carnitine to restore intracellular carnitine pools has been controversial $(6,7)$. An increased supply of carnitine may result in a further increase of long-chain acylcarnitines, compounds associated with lethal cardiac arrhythmias (8-10). 
The VLCAD knock-out (KO) mouse is an excellent model to study VLCAD deficiency (VLCADD) because it presents with similar stress-induced clinical phenotypes to humans $(5,11)$. Previous experiments have documented severe hypoglycemia and cold intolerance after fasting and a cold challenge of $8 \mathrm{~h} \mathrm{(5).} \mathrm{Intense} \mathrm{physical} \mathrm{exercise} \mathrm{is} \mathrm{characterized} \mathrm{by}$ a significantly reduced maximal running speed (5). Long-chain acylcarnitines in blood increase and free carnitine decreases in KO mice in response to various stressors. In human VLCADD, C14:0 and C14:1 acylcarnitines predominantly accumulate, whereas in VLCAD-deficient mice, the $\mathrm{C} 16$ and $\mathrm{C} 18: 1$ species are the "signature" acylcarnitines $(5,12)$. The low carnitine and high acylcarnitine concentrations in blood reflect the severity of clinical illness in mice (5). Studies on fibroblasts from patients with long-chain fatty acid oxidation disorders also document higher concentrations of long-chain acylcarnitine species in cells derived from patients with severe, early-onset phenotypes (13).

In this study, we analyzed carnitine and acylcarnitine profiles in liver, skeletal muscle (SkM), bile, and blood from wild-type (WT), heterozygous (HZ), and VLCAD KO mice under nonstressed as well as under various stress conditions to determine where acylcarnitine production takes place and whether specific stressors such as fasting and exercise selectively affect different organs. Analysis of carnitine and acylcarnitines in liver and SkM together with blood may demonstrate to what extent acylcarnitines in blood reflect tissue levels differentially and whether low blood free carnitine concentrations reflect their deficient status in tissues.

\section{METHODS}

Concentrations of carnitine and acylcarnitines in body fluids and tissues were measured in VLCAD KO mice, HZ, and WT littermates under well-fed, nonstressed conditions. Each group consisted of six mice of age 6-8wk. For determining acylcarnitine levels in liver, bile, SkM, and blood in response to stress, mice from all three genetic variants were subjected either to treadmil exercise testing or to a cold challenge at $4^{\circ} \mathrm{C}$, the latter in combination with fasting. For each stress condition, six mice of each genetic variant were used. The mice were sacrificed immediately after the stress situation was terminated. Liver and SkM (M. gastrocnemius) were removed and frozen immediately in liquid nitrogen. Blood samples were collected by heart puncture. The gallbladder was removed, and bile was collected and dried on a filter paper card.

All animal studies were performed with the approval of the Vanderbilt University Institutional Animal Care and Use Committee. The care of the animals was in accordance with the Vanderbilt University Medical Center and Institutional Animal Care and Use Committee guidelines.

Generation and genotyping of VLCAD-deficient mice. VLCAD KO mice were generated as described $(11,14)$. Genotypes were determined by duplicate PCR analyses (11)

Exercise on a treadmill. Six VLCAD KO, six HZ, and six WT mice were subjected to exercise on a treadmill equipped with an electric shock grid (2 mAmp, frequency of $4 \times / \mathrm{min}$ ). This experiment was performed as previously outlined (5). The mice had to run at $80 \%$ of their individual maximum running speed until exhaustion occurred after 45-60 min. The maximum running speed for each mouse was defined as the speed that could be maintained without falling back onto the electric shock grid for $>15 \mathrm{~s} / \mathrm{min}$. Exhaustion was defined as resting $>15 \mathrm{~s} / \mathrm{min}$ on the electric shock grid or as falling back onto the electric shock grid $>15$ times per minute.

Cold challenge with fasting. Six VLCAD KO, six HZ, and six WT mice were fasted for $8 \mathrm{~h}$ in a cold room at $4^{\circ} \mathrm{C}$ as previously reported (5). During the first $6 \mathrm{~h}$ of this fasting in cold stress, the mice were observed hourly, then every $15 \mathrm{~min}$. This experiment was terminated after $8 \mathrm{~h}$ because at that time, severe lethargy or death occurred in the VLCAD KO mice. Measurement of carnitine and acylcarnitines was performed in mice that did not die.

Analysis of carnitine and acylcarnitines. For analysis in blood and bile, carnitine and acylcarnitines were extracted with methanol from dried filter paper spots (equivalent to $25 \mu \mathrm{L}$ of blood or bile) and analyzed as their butyl esters using electrospray ionization tandem mass spectrometry as previously described $(15,16)$. Free carnitine (C0) and all even-chain C2-C24 acylcarnitines (saturated and unsaturated) were measured. Analysis of carnitine and acylcarnitines in tissues was performed according to van Vlies et al. (16a). Liver and SkM pieces of $\sim 60 \mathrm{mg}$ were lyophilized for $12 \mathrm{~h}$. The lyophilized tissues were made into a powder that was dissolved into $1 \mathrm{~mL}$ of $80 \%$ acetonitrile. After sonification and centrifugation, the supernatant was dried. As previously described $(15,16)$, carnitine and acylcarnitines were analyzed as their butyl esters by tandem mass spectrometry.

Statistical analyses. Analyses for the significance of differences were performed using $t$ test $(p<0.05)$.

\section{RESULTS}

Long-chain acylcarnitines. We were able to identify all relevant acylcarnitine species by tandem mass spectrometry after extraction from liver and SkM. Typical acylcarnitine profiles in liver and SkM are shown in Figs. 1 and 2. Mice with VLCADD accumulated the long-chain acylcarnitine species with chain lengths of 14-18 carbons in liver, SkM, blood, and bile. Under well-fed, unstressed conditions, the sum of the C14-C18 acylcarnitines was 2- to 3-fold higher in liver, SkM, and blood and 5-fold higher in bile from VLCAD KO mice, compared with WT mice (Fig. 3). The predominant acylcarnitine species that occurred in highest concentrations in WT, HZ, and $\mathrm{KO}$ mice were the $\mathrm{C} 16$ and the $\mathrm{C} 18: 1$ acylcarnitines. Despite elevated acylcarnitines in all tissues, no specific clinical phenotype was observed at 6-8 wk of age under nonstressed conditions. Later, however, nonstressed KO mice developed an increase in body weight in comparison with WT littermates under the same diet. In addition, KO mice displayed tissue steatosis, abdominal tumors, and unexpected sudden death (5).

During physical exercise, C14-C18 acylcarnitines accumulated predominantly in SkM in all WT, HZ, and KO mice. In VLCAD KO mice, these long-chain acylcarnitines reached concentrations of $\sim 200 \mathrm{nmol} / \mathrm{g}$ wet wt and were 2.5 -fold higher than under nonstressed conditions and $>6$-fold higher than

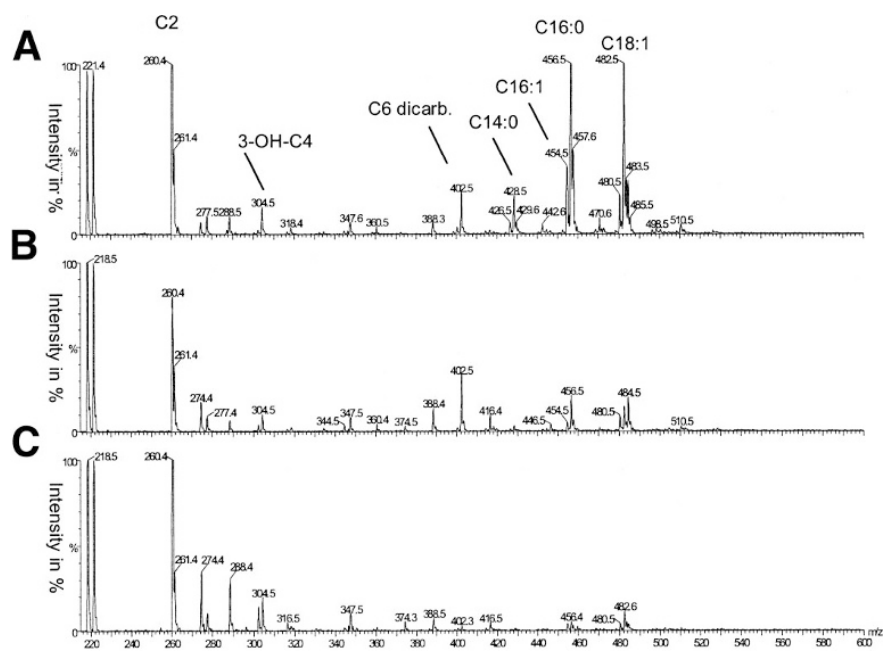

Figure 1. Acylcarnitine profiles in liver. Acylcarnitine profiles in liver from $(A)$ a single knock-out mouse after fasting at $4^{\circ} \mathrm{C},(B)$ a knock-out mouse under nonstressed conditions, and $(C)$ a wild type mouse under nonstressed conditions are shown. Concentrations are given as intensity in $\%$ of $\mathrm{d}_{3}-\mathrm{C} 0$ carnitine that has been added as an internal standard. The identification of each mass is shown in the figure. 


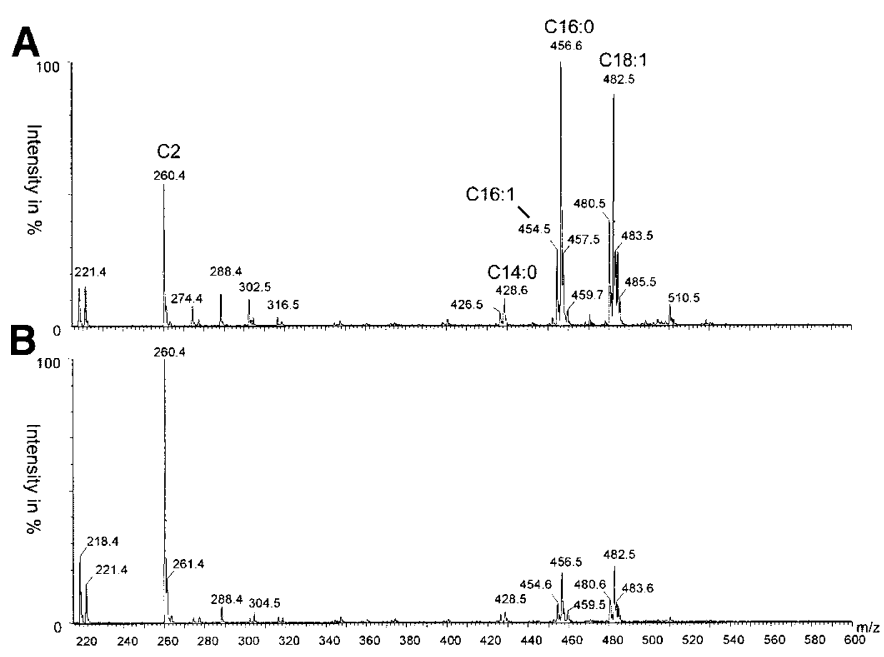

Figure 2. Acylcarnitine profiles in skeletal muscle after exercise. Acylcarnitine profiles in skeletal muscle from $(A)$ a single knock-out mouse and $(B)$ a wild type mouse after physical exercise are shown. Concentrations are given as intensity in $\%$ of $\mathrm{d}_{3}$-C0 carnitine that has been added as an internal standard. The identification of each mass is shown in the figure.

concentrations in nonstressed WT mice. This accumulation of long-chain acylcarnitines in SkM was not adequately reflected in blood (Fig. 3). In liver, no significant changes in long-chain acylcarnitine concentrations after exercise were observed. In bile, C14-C18 acylcarnitines were significantly $(p<0.05)$ reduced compared with nonstressed conditions.

After fasting in the cold at $4^{\circ} \mathrm{C}$ for $8 \mathrm{~h}, \mathrm{C} 14-\mathrm{C} 18$ acylcarnitines significantly increased in liver from WT, HZ, and KO mice, reaching concentrations of $100 \mathrm{nmol} / \mathrm{g}$ wet wt in VLCAD KO mice. These were 2-fold higher than under nonstressed conditions and $\sim 10$-fold higher than concentrations in nonstressed WT mice. These highly elevated liver acylcarnitines were well reflected in bile and blood (Fig. 3). In WT and $\mathrm{HZ}$ mice, C14-C18 acylcarnitines also significantly increased in SkM as a result of the fasting in the cold stress (Fig. $3)$.

The bile-to-blood ratios that were calculated for WT, HZ, and $\mathrm{KO}$ mice for all $\mathrm{C} 12-\mathrm{C} 18$ acylcarnitine species revealed higher concentrations in bile (Fig. 4). Bile-to-blood ratios were always higher in KO mice than in WT or HZ controls. Wellfed, nonstressed KO mice presented with bile-to-blood ratios of 10 and 30. After fasting, this ratio increased for the $\mathrm{C} 12$ acylcarnitine species to 130 (Fig. 4). The predominant longchain acylcarnitines in bile, as in tissues and blood, were the C16 and $\mathrm{C} 18$ species (Fig. 5).

Free carnitine. Free carnitine concentrations in WT mice under nonstressed conditions in liver and SkM were $~ 200-300$ nmol/g wet wt (Fig. 6). In KO mice, with a reduced blood free carnitine of $72 \%$ under normal living conditions compared with WT littermates, liver free carnitine was $400 \mathrm{nmol} / \mathrm{g}$ wet wt, significantly higher $(p<0.05)$ than in WT mice (Fig. 6).

After fasting at $4^{\circ} \mathrm{C}$, liver free carnitine significantly increased $(p<0.05)$ in all three mouse genotypes, whereas free carnitine in SkM remained unchanged. Despite stable free carnitine concentrations in tissues after fasting at $4^{\circ} \mathrm{C}$, blood free carnitine was significantly reduced in WT, HZ, and KO
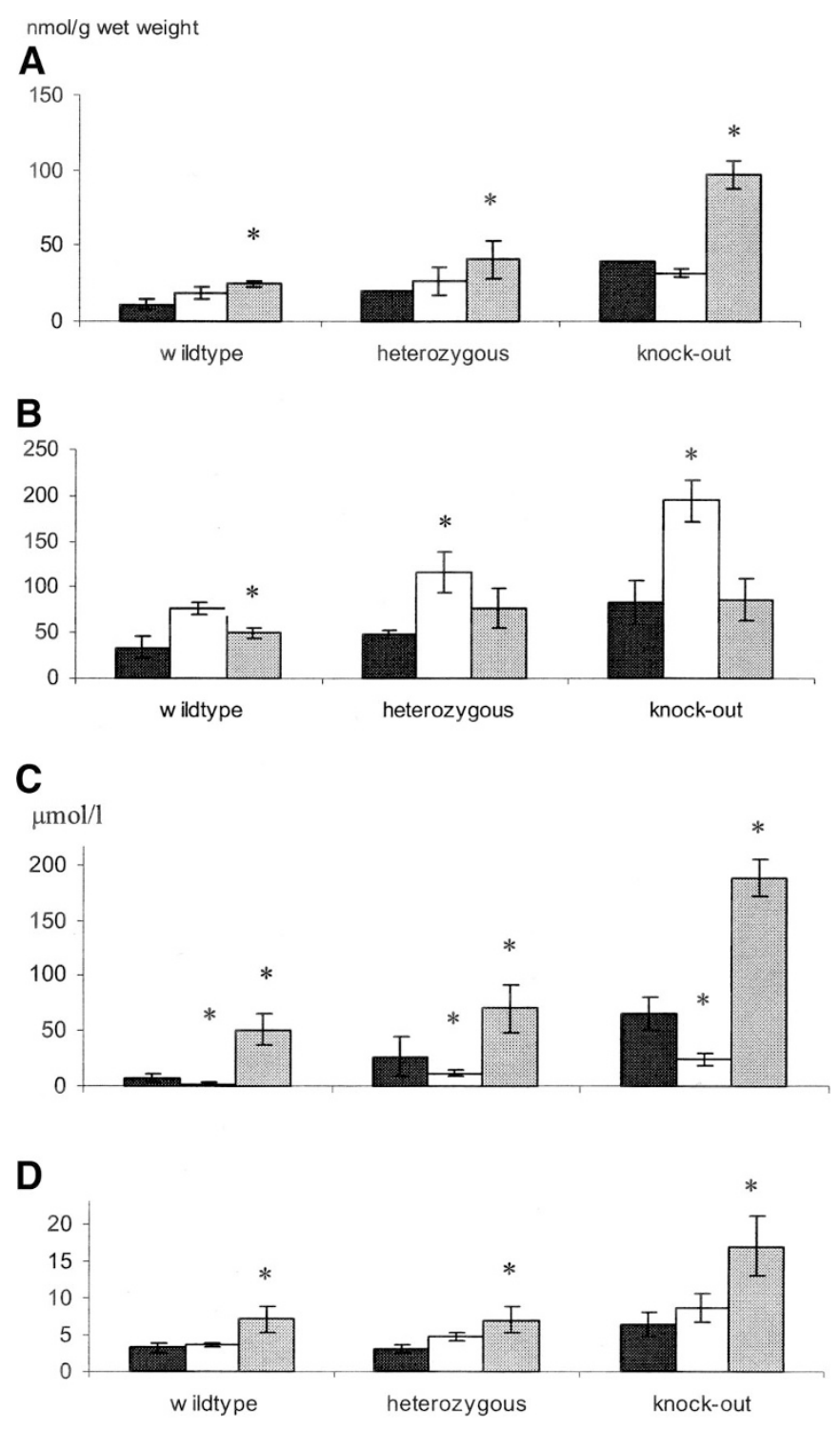

Figure 3. C14-C18 acylcarnitines in tissues and body fluids under different stress conditions. The sum of the most important long-chain acylcarnitines (C14-C18) that accumulate in VLCAD deficiency is demonstrated in liver $(A)$, skeletal muscle $(B)$, bile $(C)$ and blood $(D)$. These long-chain acylcarnitines consist of C14:2, C14:1, C14:0, C16:2, C16:1, C16:0, C18:2, C18:1, and C18:0. The three left-sided columns represent wild type mice $(n=6)$; the three middle columns heterozygous mice $(n=6)$; and the three right-sided columns knock-out mice $(n=6)$. In each mouse group, concentrations are given under nonstressed conditions (black), after intense exercise of 1 hour (white), and after an eight-hour fast in the cold (gray). The asterisks indicate significant differences $(p<0.05)$ as compared to nonstressed conditions in the respective mouse group.

mice compared with unstressed conditions and did not reflect the tissue levels (Fig. 6). The increase in liver free carnitine after fasting in the cold was not accompanied by increased bile levels.

After physical exercise, a significant decrease in free carnitine was observed in SkM of HZ and KO mice $(p<0.05)$, coinciding with a great increase of the long-chain acylcarnitines (Figs. 3 and 6). At the same time, liver free carnitine significantly increased in these two genetic mouse variants. Blood free carnitine concentrations remained unchanged, 

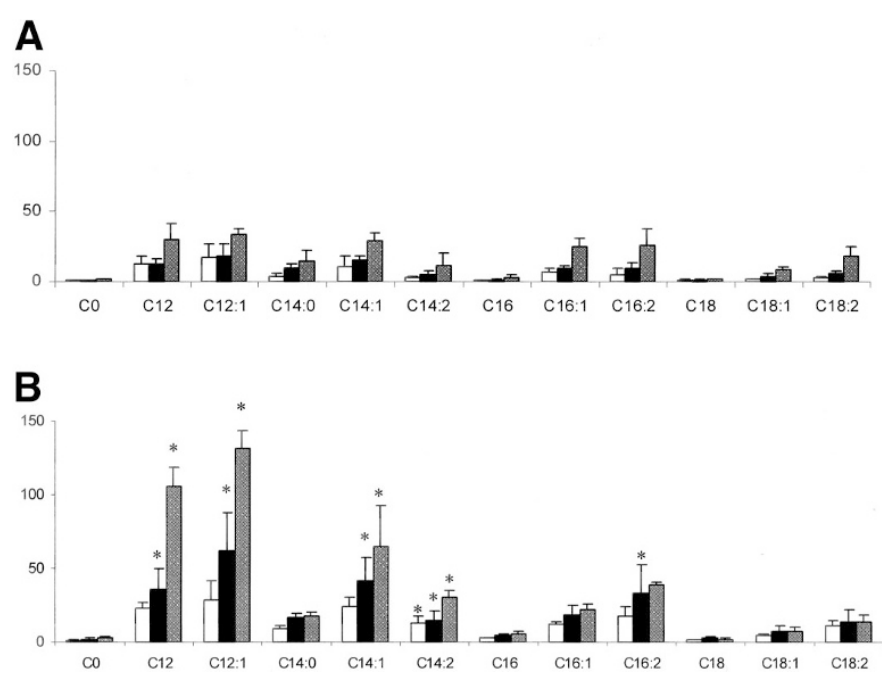

Figure 4. Bile-to-blood ratios of carnitine and acylcarnitines. Bile-to-blood ratios under nonstressed conditions $(A)$ and after fasting combined with a cold challenge $(B)$ are given for wild type mice $(\square)$, heterozygous mice ( $\square$ ), and knock-out (矅). Each mouse group consists of 6 animals. Carnitine and the $\mathrm{C} 12$ to $\mathrm{C} 18$ acylcarnitine species are demonstrated. Significant differences $(p<$ 0.05 ) between nonstressed conditions and fasting at $4^{\circ} \mathrm{C}$ are indicated by asterisks.
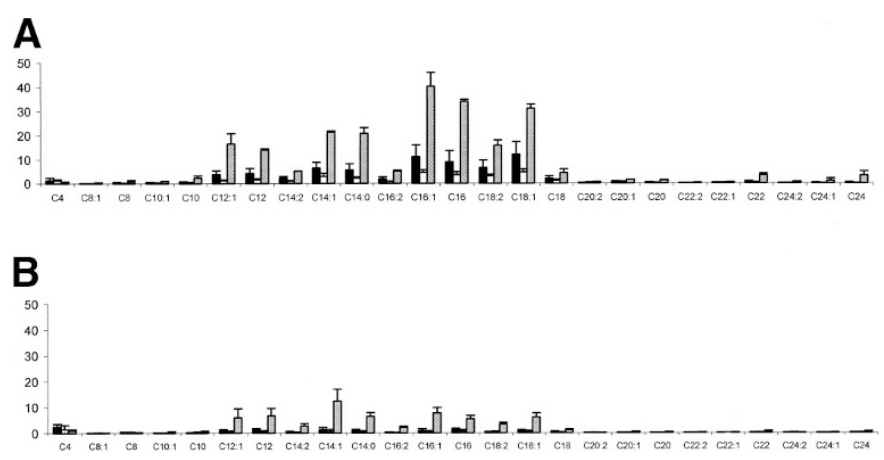

Figure 5. Acylcarnitine profiles in bile from knock-out and wild type mice. All bile acylcarnitines with an even chain length of 4 to 24 carbons from knock-out $(A)$ and wild type $(B)$ mice are shown under nonstressed conditions (black), after intense exercise (white), and after fasting combined with a cold challenge (gray). Concentrations are given in $\mu \mathrm{mol} / \mathrm{l}$ and are mean values in the different mouse groups of 6 animals. All $\mathrm{C} 12$ to $\mathrm{C} 18$ acylcarnitines species are significantly higher $(p<0.05)$ in knock-out as compared to wild type mice.

whereas in bile, a reduced level of free carnitine was observed in WT, HZ, and KO mice, together with a reduced excretion of long-chain acylcarnitines. This suggested reduced flux of carnitine and long-chain acylcarnitines into bile during exercise (Figs. 3 and 6).

\section{DISCUSSION}

This study documents increased tissue-specific long-chain acylcarnitine production in response to different stressors in VLCAD-deficient mice. Severe stress as a result of fasting in the cold significantly led to a marked induction in hepatic long-chain acylcarnitine production, as previously suggested from flux studies (17), whereas acylcarnitines in SkM accumulated predominantly during intense physical activity. The increased production of long-chain acylcarnitines in SkM from

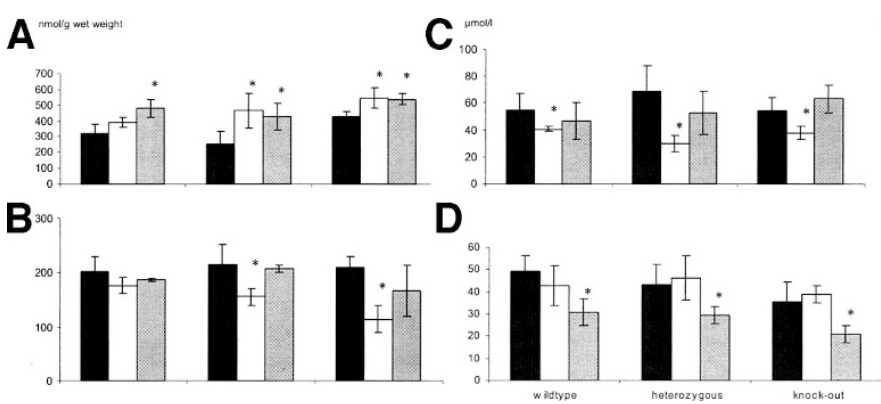

Figure 6. Free carnitine concentrations in body fluids and tissues. Mean free carnitine concentrations in liver $(A)$, skeletal muscle $(B)$, bile $(C)$ and blood $(D)$ are demonstrated. The three left-sided columns represent wild type mice $(n=$ $6)$, the three middle columns heterozygous mice $(n=6)$ and the three right-sided columns are knock-out mice $(n=6)$. In each group, concentrations are given under nonstressed conditions (black), after intense exercise of 1 hour (white), and after an eight-hour fast in the cold (gray). The asterisks indicate significant differences $(p<0.05)$ as compared to nonstressed conditions in the respective mouse group.

WT and HZ mice during fasting in the cold is likely due to an increased muscle activity needed for shivering thermogenesis that is not observed in KO mice (5). The tissue-specific acylcarnitine production that we observed in mice was associated with heterogeneous, tissue-specific phenotypes, as described in humans with VLCADD (2). Adult-onset VLCADD presents with skeletal myopathy and rhabdomyolysis that are frequently preceded and induced by intense physical exercise $(2,18,19)$, whereas in infants, reduced tolerance to fasting may allow more stress to the liver, inducing hepatic accumulation of acylcarnitines and hypoglycemia.

The elevated liver C14-C18 acylcarnitines of $100 \mathrm{nmol} / \mathrm{g}$ are closely reflected in blood and bile, suggesting that the liver efficiently catalyzes acylcarnitine export, whereas the obvious increase of acylcarnitines in SkM up to $200 \mathrm{nmol} / \mathrm{g}$ after $1 \mathrm{~h}$ of exercise is not reflected in blood. In fact, severe, episodic rhabdomyolysis in myopathic VLCADD with markedly elevated creatine kinase up to $60,000 \mathrm{U} / \mathrm{L}$ suggests muscle cell destruction that may be attributed to accumulation of longchain acylcarnitines. In contrast, the liver presents with mildly to moderately elevated transaminases during episodes of metabolic derangement but without signs of severe cell lysis (7). The exact mechanisms of how long-chain acylcarnitines might impair physiologic processes in SkM and liver are not known.

The increase in acylcarnitines in patients with VLCADD as a result of stress was accompanied by reduced free carnitine concentrations in blood, leading to the concept of "secondary carnitine deficiency." Importantly, however, our data show that changes in blood free carnitine do not correlate with carnitine homeostasis in liver and SkM. Liver free carnitine significantly increased after fasting combined with a cold challenge, and under nonstressed conditions, liver free carnitine is significantly higher in KO than in WT mice, whereas blood free carnitine is significantly lower in $\mathrm{KO}$ mice (5). These results suggest that carnitine biosynthesis in liver is induced to supply enough carnitine for conjugation with accumulating acyl-CoA. Previous studies have shown that, during an 8-h fasting period, blood free carnitine especially decreases during the first $4 \mathrm{~h}$ and then remains constant (5), suggesting an uptake of carnitine 
from blood until the increased carnitine demand can be met by carnitine biosynthesis. Studies in vegans revealed a significant positive correlation of blood carnitine levels with methionine and lysine intake, indicating that a significant portion of carnitine requirement is covered by endogenous synthesis (20). Whether an increased carnitine demand can be met by endogenous synthesis over a longer period of time needs further investigation. In contrast to the stable liver carnitine concentrations, free carnitine in skeletal muscle significantly decreased after intense exercise. Reduced free carnitine in SkM and constant blood free carnitine suggest that carnitine import from blood into SkM or carnitine biosynthesis is insufficient during $1 \mathrm{~h}$ of exercise to stabilize intramuscular carnitine levels.

It is well known that the primary site of carnitine biosynthesis is the liver (21-24). Whereas all tissues produce butyrobetaine from $\mathrm{N}^{6}$-trimethyl-lysine, the last conversion step into carnitine catalyzed by $\gamma$-butyrobetaine dioxygenase takes place only in the liver, resulting in butyrobetaine transport from other tissues via the blood to the liver for subsequent conversion (21). In fact, our data demonstrate a significant increase of free carnitine in liver after $1 \mathrm{~h}$ of exercise in $\mathrm{HZ}$ and KO mice compared with unstressed conditions, consistent with activation of liver carnitine synthesis also during physical exercise. Studies with longer exercise periods and longer follow-up investigations after exercise are necessary to confirm whether carnitine homeostasis can also be endogenously maintained in SkM. We speculate that the SkM overall is prone to carnitine import rather than acylcarnitine export in contrast to liver.

In infants with unexplained sudden death, bile has been used for analysis of acylcarnitine profiles as part of a metabolic autopsy, and 3- to 20-fold higher concentrations of long-chain acylcarnitines have been observed compared with blood $(14,25)$. In our study, the sum of the C14-C18 long-chain acylcarnitines in bile was $\sim 10$-fold higher than blood concentrations in VLCAD KO mice (Fig. 3). In contrast to tissues and blood, the $\mathrm{C} 12$ and $\mathrm{C} 14$ species in bile were present in remarkably high concentrations, and the $\mathrm{C} 12$ bile-to-blood ratio increased to 130 after prolonged fasting in the cold (Fig. 4), suggesting a nonselective excretion of all long-chain acylcarnitine species from liver to bile. The transporters and regulating mechanisms involved are not yet known.

In conclusion, tissue-specific long-chain acylcarnitine production in response to various stressors may explain the heterogeneous clinical manifestations of VLCADD in humans. Importantly, the low carnitine concentrations in blood associated with increased production and accumulation of long-chain acylcarnitines do not reflect carnitine homeostasis in tissues. In fact, free carnitine in the liver seems to be provided in sufficient amounts by carnitine biosynthesis during conditions of increased demand. Whether liver carnitine biosynthesis is sufficient to supply skeletal muscle with free carnitine during exercise needs further investigation. Our results suggest that carnitine supplementation in mitochondrial long-chain $\beta$-oxidation defects may not be required, and blood carnitine concentrations do not adequately reflect carnitine homeostasis.

\section{REFERENCES}

1. Spiekerkoetter U, Sun B, Zytkovicz T, Wanders R, Strauss AW, Wendel U 2003 MS/MS-based newborn and family screening detects asymptomatic patients with very long-chain acyl-CoA dehydrogenase deficiency. J Pediatr 143:335-342

2. Gregersen N, Andresen BS, Corydon MJ, Corydon TJ, Olsen R, Bolund L, Bross P 2001 Mutation analysis in mitochondrial fatty acid oxidation defects: exemplified by acyl-CoA dehydrogenase deficiencies, with special focus on genotype-phenotype relationship. Hum Mutat 18:169-189

3. Cox GF, Souri M, Aoyama T, Rockenmacher S, Varvogli L, Rohr F, Hashimoto T, Korson MS 1998 Reversal of severe hypertrophic cardiomyopathy and an excellent neurophysiologic outcome in very-long-chain acyl-coenzyme A dehydrogenase deficiency. J Pediatr 133:247-253

4. Brown-Harrison MC, Nada MA, Sprecher H, Vianey-Saban C, Farquhar J Jr, Gilladoga AC, Roe CR 1996 Very-long-chain acyl-CoA dehydrogenase deficiency: successful treatment of acute cardiomyopathy. Biochem Mol Med 58:59-65

5. Spiekerkoetter U, Tokunaga C, Wendel U, Mayatepek E, Duran M, Wijburg FA, Wanders RJ, Strauss AW 2004 Changes in blood carnitine and acylcarnitine profiles of very long-chain acyl-CoA dehydrogenase-deficient mice subjected to stress. Eur J Clin Invest 34:191-196

6. Touma EH, Rashed MS, Vianey-Saban C, Sakr A, Divry P, Gregersen N, Andresen BS 2001 A severe genotype with favourable outcome in very long chain acyl-CoA dehydrogenase deficiency. Arch Dis Child 84:58-60

7. Spiekerkotter U, Schwahn B, Korall H, Trefz FK, Andresen BS, Wendel U 2000 Very-long-chain acyl-coenzyme A dehydrogenase (VLCAD) deficiency: monitoring of treatment by carnitine/acylcarnitine analysis in blood spots. Acta Paediatr 89:492495

8. Pauly DF, Pepine CJ 2003 The role of carnitine in myocardial dysfunction. Am J Kidney Dis 41:S35-S43

9. Tein I 2002 Role of carnitine and fatty acid oxidation and its effects in infantile epilepsy. J Child Neurol 17:57-82

10. Bonnet D, Martin D, de Lonlay P, Villain E, Jouvet P, Rabier D, Brivet M, Saudubray JM 1999 Arrhythmias and conduction defects as presenting symptoms of fatty acid oxidation disorders in children. Circulation 100:2248-2253

11. Exil VJ, Roberts R, Sims H, McLaughlin JE, Malkin RA, Gardner CD, Ni G, Rottman JN, Strauss AW 2003 Very-long-chain acyl-coenzyme A dehydrogenase deficiency in mice. Circ Res 93:448-455

12. Cox KB, Hamm DA, Millington DS, Matern D, Vockley J, Rinaldo P, Pinkert CA, Rhead WJ, Lindsey JR, Wood PA 2001 Gestational, pathologic and biochemical differences between very-long-chain acyl-CoA dehydrogenase deficiency and longchain acyl-CoA dehydrogenase deficiency in the mouse. Hum Mol Genet 10:20692077

13. Giak Sim K, Carpenter K, Hammond J, Christodoulou J, Wilcken B 2002 Quantitative fibroblast acylcarnitine profiles in mitochondrial fatty acid $\beta$-oxidation defects: phenotype/metabolite correlations. Mol Genet Metab 76:327-334

14. Papaioannou VE, Johnson RS 1993 Gene Targeting: A Practical Approach. Oxford University Press, Oxford, pp 107-146

15. Vreken $\mathrm{P}$, van Lint AE, Bootsma AH, Overmars H, Wanders RJ, van Gennip AH 1999 Quantitative plasma acylcarnitine analysis using electrospray tandem mass spectrometry for the diagnosis of organic acidemias and fatty acid oxidation defects. J Inherit Metab Dis 22:302-306

16. Chace DH, DiPerna JC, Mitchell BL, Sgroi B, Hofman LF, Naylor EW 2001 Electrospray tandem mass spectrometry for analysis of acylcarnitines in dried postmortem blood specimens collected at autopsy from infants with unexplained cause of death. Clin Chem 47:1166-1182

16a. van Vlies N, Tain L, Overmars H, Bootsma AH, Kulik W, Wanders RJA, Wood PA, Vaz FM 2004 Characterization of carnitine and fatty acid metabolism in the long-chain acyl-CoA dehydrogenase deficient mouse. Biochem J epub ahead of print

17. Sandor A, Cseko J, Kispal G, Alkonyi I 1990 Surplus acylcarnitines in the plasma of starved rats derive from the liver. J Biol Chem 265:22313-22316

18. Ogilvie I, Pourfazam M, Jackson S, Stockdale C, Bartlett K, Turnbull DM 1994 Very long-chain acyl coenzyme A dehydrogenase deficiency presenting with exerciseinduced myoglobinuria. Neurology 44:467-473

19. Scholte HR, Van Coster RN, de Jonge PC, Poorthuis BJ, Jeneson JA, Andresen BS, Gregersen N, de Klerk JB, Busch HF 1999 Myopathy in very-long-chain acyl-CoA dehydrogenase deficiency: clinical and biochemical differences with the fatal cardiac phenotype. Neuromuscul Disord 9:313-319

20. Krajcovicova-Kudlackova M, Simoncic R, Bederova A, Babinska K, Beder I 2000 Correlation of carnitine levels to methionine and lysine intake. Physiol Res 49:399402

21. Tanphaichitr V, Broquist HP 1974 Site of carnitine biosynthesis in the rat. J Nutr 104:1669-1673

22. Cox RA, Hoppel CL 1974 Carnitine and trimethylaminobutyrate synthesis in rat tissues. Biochem J 142:699-701

23. Haigler HT, Broquist HP 1974 Carnitine synthesis in rat tissues slices. Biochem Biophys Res Commun 56:676-681

24. Vaz FM, Wanders RJ 2002 Carnitine biosynthesis in mammals. Biochem J 361:417429

25. Rashed MS, Ozand PT, Bennett MJ, Barnard JJ, Govindaraju DR, Rinaldo P 1995 Inborn errors of metabolism diagnosed in sudden death cases by acylcarnitine analysis of postmortem bile. Clin Chem 41:1109-1114 